\title{
Cost/benefit and the effect of sample preservation procedures on quantitative patterns in benthic ecology
}

\author{
Gabriel Barros Gonçalves de Souza ${ }^{1 *}$ (i) and Francisco Barros ${ }^{2}$
}

\begin{abstract}
Some benthic assemblages studies have tested the effects of different preservation procedures on biomass, but their influence on quantitative patterns (number of species and abundance) is still unclear. We evaluated the influence of two sample preservation procedures on quantitative patterns in benthic ecology. Ten sampling points were systematically interspersed on two types of sediment (sandy and muddy). At each sediment type, samples from five sampling points were fixed in 10\% formalin, and the other five points were preserved in $70 \%$ ethanol (without previous fixation). Three replicates were collected at each sampling point, and samples were washed in 0.5 mesh size and sorted in laboratory. A cost/benefit analysis was performed considering the washing time in laboratory and the costs of substances. A total of 1970 individuals were collected (muddy sediment: 132; sandy sediment: 1838), belonging to 121 taxa (muddy: 49; sandy: 83). Assemblages preserved in ethanol were composed of 795 individuals and 80 taxa, while those fixed with formalin had 1173 individuals and 94 taxa. Polychaeta predominated as the most abundant group for both preservation procedures. For the whole benthic community, significant differences occurred only between sediment types. Significant differences in the number of individuals of polychaetes were observed for the different preservation procedures in sandy sediment. Ethanol has the best cost/benefit ratio in both sediment types due to additional costs to attend safety requirements for formalin-fixed samples. Further studies should evaluate how quantitative patterns are affected by exposure time of preservation, anesthesia interaction, and morphological deformations (e.g. impossibility of identification).
\end{abstract}

Keywords: Macrobenthos, Benthic methods, Soft bottom, Ethanol, Formalin, Cost/benefit ratio

\section{Background}

Soft bottom benthic macrofaunal assemblages are composed of several groups of invertebrates, and the most abundant groups are often polychaetes, crustaceans, and mollusks [e.g. 1]. The most appropriate sampling and preservation procedures for these organisms have been described in the literature [e.g. 2, 3], and different substances for anesthesia, fixation, and preservation were suggested for each taxonomic group [2]. However, in

\footnotetext{
*Correspondence: gabrielbbarros@gmail.com

1 Programa de Pós-Graduação em Ecologia, Laboratório de Biologia e Tecnologia Pesqueira, Departamento de Biologia Marinha, Centro de Ciências da Saúde, Instituto de Biologia, Universidade Federal do Rio de Janeiro, Av. Carlos Chagas Filho, 373, Bloco A, Ilha do Fundão, Rio de Janeiro, RJ CEP 21944-970, Brazil

Full list of author information is available at the end of the article
}

benthic assemblage studies the entire biological sample is stored using the same substance, since separating the groups by using specific preservation procedures would be, in the best scenario, extremely labor intensive. The commonly used sample preservation procedures are: (1) fixation in $4-10 \%$ formalin and subsequent preservation in $70 \%$ ethanol; or (2) simply preservation in $70 \%$ ethanol, without use of fixative substance [4].

Although $10 \%$ formalin (4\% formaldehyde) is one of the most commonly used substances for fixing benthic invertebrates, its use requires some precautions. To avoid osmotic imbalances in marine organisms, dilution of formalin into seawater is advised [2]. Furthermore, formalin solutions usually get oxidized to form formic acid [5], which can cause deformation to the specimens if stored by long periods of time. In that case, to neutralize the 
substance, borax (sodium borate) or hexamine (hexamethylenetetramine) are frequently added [3]. In addition, formalin contains toxic compounds that have carcinogenic effects [e.g. 6, 7], and special care is required to handle samples containing this substance.

Due to the problems mentioned above, some researchers prefer to use only ethanol (a less toxic substance) to preserve the samples collected. However, the use of this substance to preserve samples in field presents some disadvantages, mainly due to its volatility [2]. There is a precipitate formation when the ethanol is mixed with seawater, which can also cause the separation of lamellibranch molluscs from their shells [3].

Studies evaluating the influence of different preservation procedures on benthic invertebrates are scarce. Most of the researches published to date have analyzed the effects of these procedures on biomass estimates of benthic organisms [e.g. 8-13]. These studies have generally analyzed the effect of these procedures on a few species. Few studies have examined the influence of the preservative substances on the morphological characters of invertebrates [14]. Furthermore, there are no studies evaluating potential losses of benthic material and/ or the impossibility of identification due to preservation procedures.

In the present study we evaluated the influence of two sample preservation procedures, with and without formalin fixation, on benthic data in sandy and muddy sediments. Univariate quantitative patterns (number of taxa and individuals) and multivariate patterns (assemblage structure) were analyzed. We also tested potential differences for polychaetes separately, since this group has a relatively fragile body and the direct storage of this taxon in ethanol may cause deformations in morphological structures [see 2].

\section{Methods}

\section{Study area}

The present study was carried out in Todos os Santos Bay (TSB), which is the second largest Brazilian coastal bay with an area of approximately $1233 \mathrm{~km}^{2}$ [15]. There are more than three million people and several large port terminals around this bay. Despite the strong human impact the TSB still has significant extension of coral reefs, mangroves, and estuaries. Sampling was performed in the shallow infralittoral of two sampling sites: in a muddy sediment area situated in front of Inema Beach $\left(12^{\circ} 49^{\prime} 21.9^{\prime \prime} \mathrm{S}\right.$ and $\left.30^{\circ} 29^{\prime} 34.1^{\prime \prime} \mathrm{W}\right)$, and in a sandy sediment patch close to Ribeira Beach $\left(12^{\circ} 53^{\prime} 51.5^{\prime \prime} \mathrm{S}\right.$ and $\left.38^{\circ} 30^{\prime} 11.5^{\prime \prime} \mathrm{W}\right)$. Inema is located close to Aratu Bay, and the sampled area presented sediments with predominance of fines (silt and clay) and average depth of six meters. The sampling site close to Ribeira is between the Salvador channel and the Itapagipe Bay, and the sediments were predominantly sandy (large amount of carbonate biodetrites) with average depth of five meters.

\section{Sampling procedures}

Sampling was performed in May 2009. In each sediment type, we set 10 sampling points systematically interspersed [see 16], at a distance of 10 meters from each other. These sampling points were interspersed according to the sample preservation procedure, totaling five points for each one (Fig. 1). We chose this sampling design mainly based in two aspects: (1) independence of treatments (preservation procedures), which was necessary for the used inferential analysis (ANOVA); and (2) the influence of small-scale variability (patches). As stated by Hurlbert [16], a systematic interspersed design is preferable to a randomized one especially if the spacing interval does not coincide with some periodically varying property of the experimental area. In that case, we restricted the sampling area to avoid sediment patches (which is a real problem in randomly sampling) and we ensure that there was no undesirable difference in the sampled area (troughs/ripples, wrack piles, etc.).

At each sampling point, divers haphazardly collected three sediment samples using PVC (Polyvinyl chloride) corers $\left(15 \mathrm{~cm}\right.$ depth/10 $\mathrm{cm}$ diameter/0.008 $\mathrm{m}^{2} / 1.2 \mathrm{~L}$ ). Each benthic sample was separately washed in situ through $0.5 \mathrm{~mm}$ mesh size and stored in properly labeled plastic bags containing the respective fixing substance (ethanol or formalin). The $70 \%$ ethanol was prepared in the laboratory by adding $370 \mathrm{~mL}$ of water to $1 \mathrm{~L}$ of $96^{\circ}$ GL ethanol (not methylated), which provided a total of $1.37 \mathrm{~L}$ of $70 \%$ ethanol per each original concentration bottle. Formalin was diluted in seawater, in the fieldwork, to avoid osmotic unbalances in organisms. Each $100 \mathrm{~mL}$ of $37 \%$ formaldehyde was diluted into $900 \mathrm{~mL}$ of seawater to reach $1 \mathrm{~L}$ of $10 \%$ formalin. Samples were stored in a laboratory freezer to minimize the volatility, and sieving started 20 days after sampling. Samples containing formalin or ethanol were washed again to remove the fixing substance and sorted using a stereoscopic microscope. To avoid possible differences due the storage time of samples, we analyzed the same amount of ethanol and formalin preserved samples each sieving day during almost 1 month. The $10 \%$ formalin-fixed samples were washed in a laboratory fume hood, using all necessary personal protective equipment-PPE (lab coat, mask, safety glasses, gloves). All specimens were identified to lowest possible taxonomic level (i.e. species or morphotypes) and later preserved in $70 \%$ ethanol. 


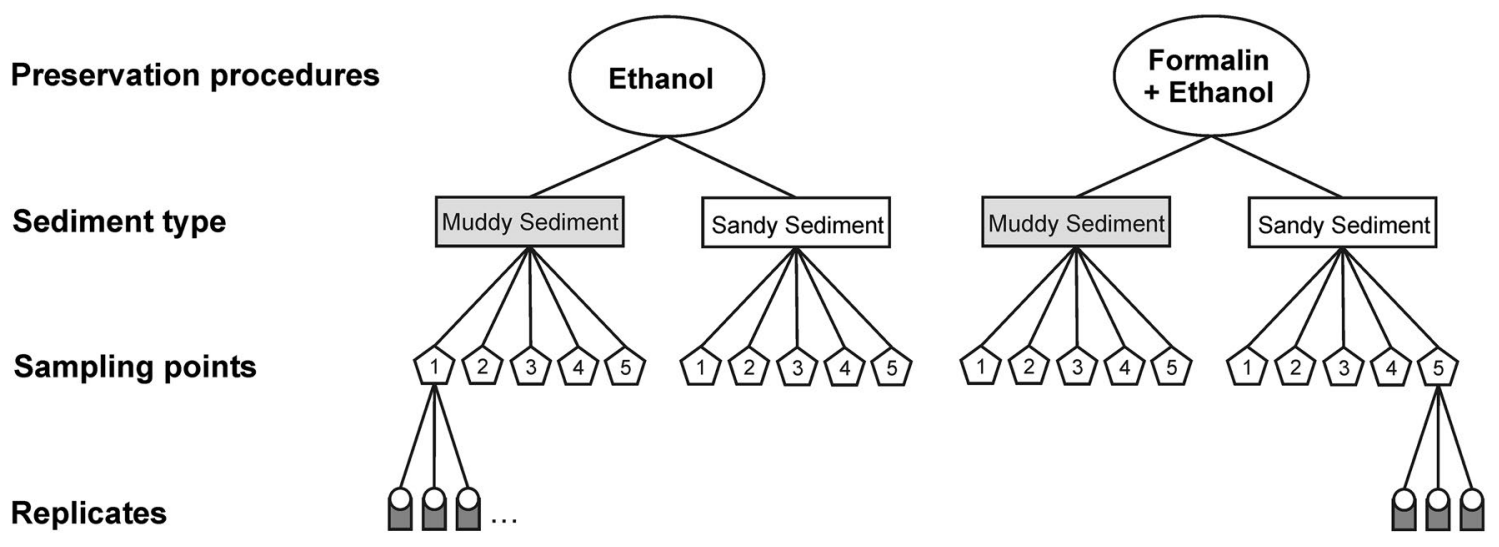

* Sampling points systematically interspersed

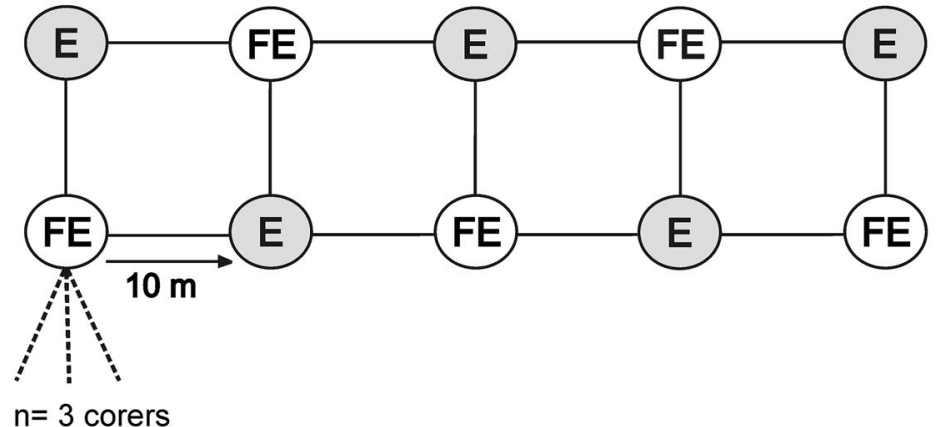

Fig. 1 Schematic overview of the systematic interspersed sampling design applied in both sites. E ethanol; FE formalin and ethanol

\section{Data analysis}

We performed a three-way ANOVA to test the effect of different preservation procedures on the number of taxa and number of individuals for the whole benthic assemblage, and for the polychaetes assemblage (usually the most abundant taxa). Factors in the analysis were: (1) preservation procedure (fixed and orthogonal with two levels: ethanol and formalin + ethanol); (2) sediment type (fixed and orthogonal with two levels: sandy and muddy); and (3) sampling points (random and nested with five levels). Software GMAV5 for Windows (Institute of Marine Ecology, University of Sydney) was used and corer sediment samples were not pooled for each sampling point in this analysis. Cochran's Test verified the homogeneity of variances. The Student Newman-Keuls (SNK) a posteriori test was used to assess significant differences. We tested the effect of sampling procedures in multivariate patterns using a non-parametric multivariate analysis of variance (PERMANOVA; Permanova 6 software) [17]. This procedure is considered a good alternative to the traditional MANOVA problems (e.g. assumption of multinormality) since it uses permutation methods to calculate $p$ values [18].

Non-metric multidimensional scaling (nMDS, software PRIMER 6) ordinations, based on using a Bray-Curtis, were performed to visualize potential difference in similarities of benthic assemblage due to sample preservation procedures (corer sediments samples pooled per sampling point). We added a dummy variable (1 to all samples) to include samples that had no specimens collected.

A cost/benefit analysis was performed using the index proposed by Souza and Barros [19] with the formula: $\mathrm{CB}=\left(C_{t} /(1-p)\right) / 1000$; in which $C t$ is the total cost, and $p$ is the precision. The costs $(C t)$ were calculated using the formula indicated by Andrew and Mapstone [20]: $C_{t}=n \cdot C_{u}+C_{a}$, in which $n$ is the number of sampling points for each procedure (corer sediment samples pooled for each point), $C_{u}$ is the cost of each sampling unit, measured from the mean time taken to wash samples in laboratory, and $C_{a}$ is the additional cost, assumed as spent value to purchase substances for the preservation of samples in the field, and for safety equipment. We 
converted the time needed to wash the samples in laboratory into monetary value using the fee suggested by the 'Conselho Federal de Biologia-CFBio' (Federal Council of Biology), which is R \$ 90/h (Brazilian Real) for master degree professionals. The monetary value was converted to U.S. dollars using the current exchange rate (May 10th, 2017). The precision $(p)$ was calculated using the formula $p=S E / X$ (which $S E=(s / \sqrt{ } n) / X$ ); where $S E$ is the standard error estimated from the standard deviation $(s)$ for a given sample size $(n)$, and $(X)$ is the sample mean. The sample size $(n)$ was the number of sampling points for each preservation procedure in each sediment type $(n=5)$, and the mean and standard deviation were calculated from the sum of abundances at each point.

\section{Results}

\section{Benthic macrofauna}

A total of 1968 individuals (131 in muddy sediment and 1837 in sandy sediment) were collected, comprising 119 taxa (48 in mud and 82 in sand) belonging to eight phyla. Polychaeta was the most diverse taxon (39\%), followed by Mollusca (28\%) and Crustacea (28\%). We also observed specimens belonging to Nemertea, Sipuncula, Echinodermata, and Cephalochordata groups. Polychaetes were likewise the most abundant organisms ( $46 \%$ of the total number of individuals), followed by Sipuncula (25\%). At the sandy sediment, a morphotype of Sipuncula (26.8\%) prevailed as the most abundant taxon, followed by Exogone sp. (15.3\%) and Branchiostoma caribaeum (13.4\%). The polychaete Lumbrinereis sp. (12.1\%) was the most abundant in the muddy sediment, followed by Paraprionospio sp. (9.8\%) and Olivella minuta (9.8\%).

\section{Preservation procedures}

Samples only preserved with $70 \%$ ethanol were composed of a total of 795 individuals and 80 taxa, while in those fixed with formalin 10\% we found 1173 individuals and 94 taxa. Despite this numerical difference, the dominance patterns among groups were the same for the two methods. Polychaeta was the most abundant group for the two preservation procedures, followed by Mollusca and Crustacea (Fig. 2).

In the muddy sediment, a total of 52 individuals and 27 taxa were found in ethanol preserved samples, while 79 individuals and 35 taxa were found in formalin fixed samples. On the other hand, in the sandy sediment, we counted 743 individuals and 56 taxa in ethanol preserved samples, and 1094 individuals and 62 taxa in formalin fixed samples. Despite this difference in the absolute value, statistical differences between preservation procedures were not observed.

ANOVA revealed significant differences only between sediment types and sampling points $(p<0.01)$ for the number of taxa and individuals of the whole macrofaunal assemblage (Table 1). The difference between sediment types is due to the sandy site presents a higher number of taxa and individuals than muddy site. Regarding the sampling points, one sampling point in the sandy site presented high values in the number of individuals of the most abundant taxa, as well as a higher number of taxa (SNK result). In the Analysis of Variance performed with the polychaetes assemblage, we observed significant differences in the number of individuals between the preservation procedures $(\mathrm{p}<0.025)$. However, this difference was only observed on the sandy sediment. Significant differences were also observed between sediment types and sampling points $(\mathrm{p}<0.01)$ for abundance and number of taxa (see Table 1). Additionally, significant differences were not observed for the most abundant taxa.

In the non-parametric multivariate analysis of variance (PERMANOVA), only sediment types presented significant differences $(\mathrm{p}<0.01)$, for both macrofaunal and polychaete assemblages (Table 2). This difference is due to the same observed in the univariate tests, since the sandy site presented a higher number of taxa and individuals than the muddy site. In the nMDS ordinations (stress $=0.11)$, no clear separation between the treatments (ethanol and formalin) was observed (Fig. 3). Due to the high difference in faunal composition between the muddy and sandy assemblages, separated ordinations were also conducted for each site.

\section{Cost/benefit analysis}

Separated cost/benefit analyzes were performed for each sediment type due to the large difference in faunistic structure. In the muddy sediment, samples fixed in formalin presented slightly better precision (0.14) than those in ethanol (0.19). However, for the sandy sediment, samples fixed in formalin showed quite lower precision (0.16) than those only preserved in ethanol (0.04) (see Table 3). Samples washing time in laboratory were similar between the preservation procedures and ranged from 4 to $18 \mathrm{~min}$. Ethanol and formalin samples demanded on average (considering both sediment types) $7.5 \mathrm{~min}( \pm 1.9)$ and $8.1 \mathrm{~min}( \pm 2.8)$, respectively. Consequently, the cost of each sampling unit was similar between preservation procedures. In terms of additional costs, the price of $1 \mathrm{~L}$ of $96^{\circ} \mathrm{GL}$ ethanol is about $\mathrm{R} \$ 10$ (\$3.14), and with each liter it is possible to make approximately $1.5 \mathrm{~L}$ of $70 \%$ ethanol. Almost $10 \mathrm{~L}$ of $10 \%$ formalin can be produced with $1 \mathrm{~L}$ of $37 \%$ formaldehyde $(\mathrm{R} \$ 18$ per liter $=\$ 5.66)$. Thus, the costs of purchase substances where calculated considering that it is possible to preserve four corer sediment samples with $1 \mathrm{~L}$ of each substance. Furthermore, the cost of safety equipment 

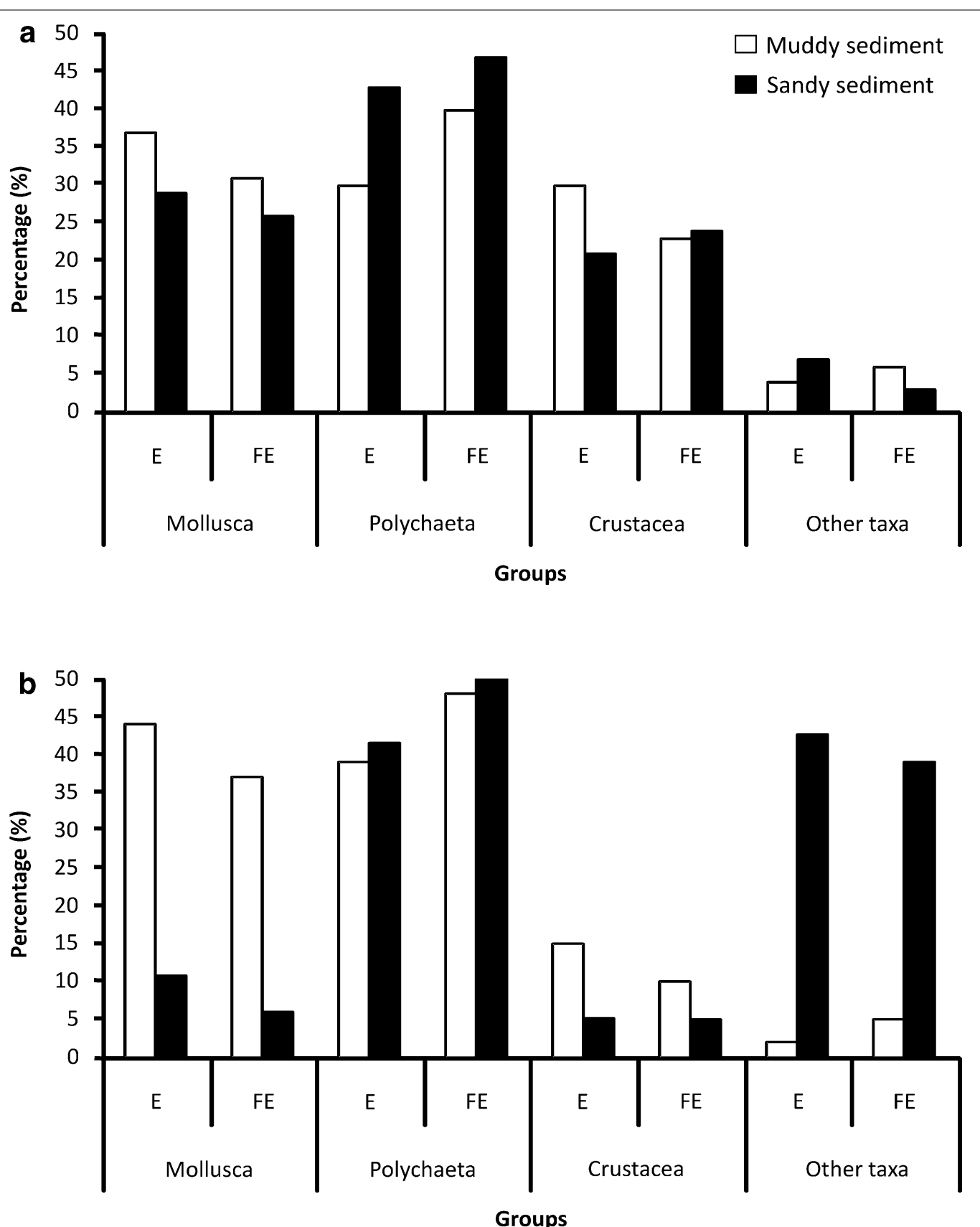

Fig. 2 Number of taxa (a) and individuals (b) of benthic groups in muddy and sandy sediments. E ethanol; FE formalin and ethanol

as masks ( $\mathrm{R} \$ 25=\$ 7.86)$, safety glasses $(\mathrm{R} \$ 5=\$ 1.57)$, and fume hood (about $\mathrm{R} \$ 3000=\$ 943.4$ ) where added to the analysis of formalin-fixed samples. It caused a high difference between the additional costs of the substances mainly due to high cost of acquisition and installation of fume hood. In this case, the cost/benefit ratio of the preservation procedures was quite different in both sediment types, and ethanol showed much better cost/benefit ratio than formalin (Fig. 4a). Nonetheless, we took in consideration that some labs already have a fume hood installed, turning this cost irrelevant. Thus, the cost/benefit ratio was also calculated excluding the fume hood costs, showing a slightly difference between preservation procedures (especially in the muddy sediment). For this analysis, ethanol still has the best cost/ benefit ratio (Fig. 4b). 
Table 1 ANOVA results analyzing the number of taxa and individuals of benthic assemblage and polychaetes assemblage

\begin{tabular}{|c|c|c|c|c|c|c|c|}
\hline \multirow[t]{2}{*}{ Factors } & \multirow[t]{2}{*}{$D F$} & \multicolumn{3}{|c|}{ Number of individuals } & \multicolumn{3}{|c|}{ Number of taxa } \\
\hline & & MS & $F$ & $p$ & MS & $\mathbf{F}$ & $p$ \\
\hline \multicolumn{8}{|l|}{ Macrofauna } \\
\hline Preservation (Pr) & 1 & 2356.27 & 4.26 & $0.0555 \mathrm{NS}$ & 25.35 & 2.65 & $0.1234 \mathrm{NS}$ \\
\hline Sediment (S) & 1 & $48,507.27$ & 87.77 & $0.0001^{*}$ & 1848.15 & 192.85 & $0.0001^{*}$ \\
\hline $\operatorname{Pr} \times S$ & 1 & 1749.60 & 3.17 & 0.0942 NS & 0.82 & 0.09 & $0.7741 \mathrm{NS}$ \\
\hline Sampling points $(\operatorname{Pr} \times S)$ & 16 & 552.66 & 8.53 & $0.0001^{*}$ & 9.58 & 2.53 & $0.0087^{*}$ \\
\hline Residual & 40 & 64.77 & & & 3.78 & & \\
\hline \multicolumn{8}{|l|}{ Polychaetes } \\
\hline Preservation (Pr) & 1 & 1058.40 & 7.16 & $0.0166^{*}$ & 19.27 & 2.57 & $0.1281 \mathrm{NS}$ \\
\hline Sediment (S) & 1 & $10,454.40$ & 70.72 & $0.0001^{*}$ & 589.07 & 78.72 & $0.0001^{*}$ \\
\hline $\operatorname{Pr} \times S$ & 1 & 777.60 & 5.26 & 0.0357 NS & 0.27 & 0.04 & $0.8526 \mathrm{NS}$ \\
\hline Sampling points $(\operatorname{Pr} \times S)$ & 16 & 147.82 & 4.62 & $0.0001^{*}$ & 7.48 & 3.40 & $0.0001^{*}$ \\
\hline Residual & 40 & 31.98 & & & & 2.20 & \\
\hline
\end{tabular}

NS not significant, $D F$ degrees of freedom, MS mean squares

* Significant difference for $a=0.05$

Table 2 PERMANOVA results analyzing the benthic assemblage and polychaetes assemblage from muddy and sandy sediment

\begin{tabular}{|c|c|c|c|c|c|c|}
\hline Factors & $D F$ & MS & $F$ & Unique permutations & $p($ perm $)$ & $p(\mathrm{MC})$ \\
\hline \multicolumn{7}{|l|}{ Macrofauna } \\
\hline Preservation (Pr) & 1 & 4598.62 & 1.58 & 9929 & 0.1793 NS & 0.1238 NS \\
\hline Sediment (S) & 1 & $72,874.61$ & 2.10 & 9907 & $0.0001^{*}$ & $0.0001^{*}$ \\
\hline $\operatorname{Pr} \times S$ & 1 & 4484.29 & 1.54 & 9913 & 0.1814 NS & 0.1335 NS \\
\hline Sampling points $(\operatorname{Pr} \times S)$ & 16 & 2903.83 & 1.20 & 9800 & 0.1022 NS & 0.1144 NS \\
\hline Residual & 40 & 2426.04 & & & & \\
\hline \multicolumn{7}{|l|}{ Polychaetes } \\
\hline Preservation (Pr) & 1 & 5218.38 & 1.71 & 9928 & 0.1683 NS & 0.1159 NS \\
\hline Sediment (S) & 1 & $71,914.60$ & 23.60 & 9921 & $0.0001^{*}$ & $0.0001^{*}$ \\
\hline $\operatorname{Pr} \times S$ & 1 & 5420.79 & 1.78 & 9927 & 0.1654 NS & 0.1005 NS \\
\hline Sampling points $(\operatorname{Pr} \times S)$ & 16 & 3046.89 & 1.30 & 9840 & 0.0502 NS & 0.0545 NS \\
\hline Residual & 40 & 2344.74 & & & & \\
\hline
\end{tabular}

NS not significant, DF degrees of freedom, MS mean squares, $p($ perm) permuted $p$ value, $p(M C)$ Monte Carlo $p$ value

* Significant difference for $a=0.05$

\section{Discussion}

Studies that have evaluated the effect of different biological sample preservation procedures have focused essentially on changes in biomass estimation values. However, there is a contradiction in these studies, since some studies do not recommend using $70 \%$ ethanol because of the loss of more weight than formalin-preserved specimens, and other studies do not confirm this observation [see 13]. We did not find significant differences in the quantitative patterns (uni and multivariate) for the macrofaunal assemblage. This is similar to some previous studies that did not observe differences in the influence of formalin and ethanol on the benthic assemblage biomass [11] and on some benthic species biomass [12,13].

The effect of the preservative substance on biomass estimation as a function of the exposure time of the sample is another aspect usually evaluated in previous studies. Some researchers have observed that there is higher weight loss of the specimens in the first few weeks, and there is no difference between samples kept in formalin or ethanol [e.g. 11, 13]. Nevertheless, there is no information about the effect of storage time of samples on quantitative patterns (i.e. richness and abundance). In the present study, we avoid the possible effect of storage time 


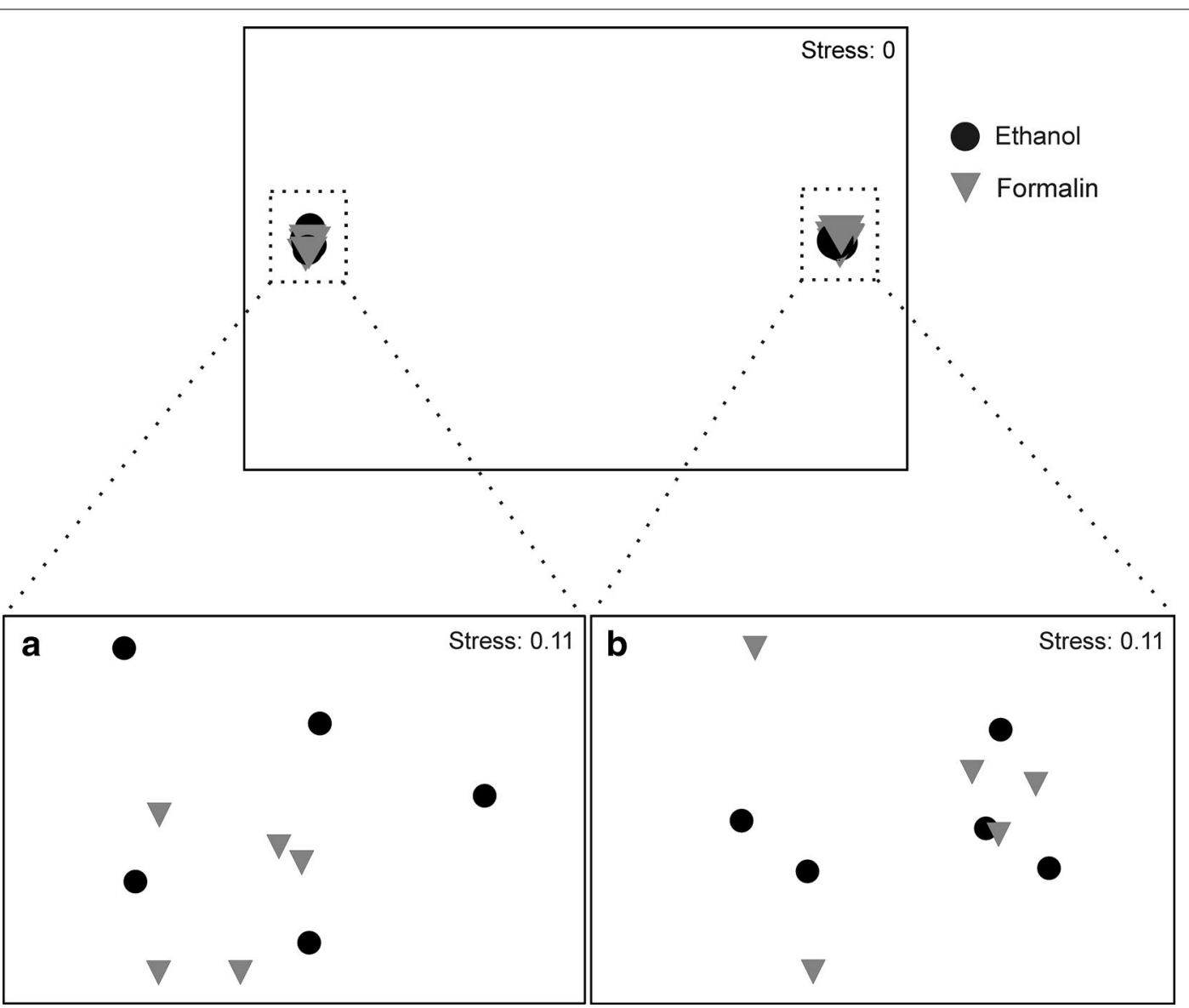

Fig. 3 nMDS ordinations of benthic assemblage sampled in $\mathbf{a}$ muddy sediment and $\mathbf{b}$ sandy sediment

Table 3 Precision, mean washing time, and costs of the preservation procedures analyzed

\begin{tabular}{|c|c|c|c|c|}
\hline & \multicolumn{2}{|c|}{ Muddy sediment } & \multicolumn{2}{|c|}{ Sandy sediment } \\
\hline & Ethanol & Formalin & Ethanol & Formalin \\
\hline Precision & 0.19 & 0.14 & 0.04 & 0.16 \\
\hline Mean washing time (min) & $7.1( \pm 1.6)$ & $7.5( \pm 3.5)$ & $8.0( \pm 2.0)$ & $8.7( \pm 1.8)$ \\
\hline Mean cost of sampling unit (U.S. Dollar) & $\$ 10$ & $\$ 10.7$ & $\$ 11.3$ & $\$ 12.4$ \\
\hline Additional costs (U.S. Dollar) & $\$ 9.4$ & $\begin{array}{l}2.83=\text { formaldehyde } \\
7.86=\text { mask } \\
1.57=\text { safety glasses } \\
943.4=\text { fume hood } \\
\text { Total: } \$ 955.7\end{array}$ & $\$ 9.4$ & $\begin{array}{l}2.83=\text { formaldehyde } \\
7.86=\text { mask } \\
1.57=\text { safety glasses } \\
943.4=\text { fume hood } \\
\text { Total: } \$ 955.7\end{array}$ \\
\hline
\end{tabular}

by sieving the same amount of ethanol and formalin preserved samples per sieving day.

As for most zoological groups, the fixation in $5-10 \%$ formaldehyde has been suggested for Polychaeta, Mollusca, and Crustacea, the most abundant macrofauna groups of soft bottom [2]. Although we did not quantify the degree of conservation of the organisms, we observed that $10 \%$ formalin-fixed polychaetes had an apparent better degree of conservation (more rigid and intact structures) than those only preserved with $70 \%$ ethanol (some with a pasty appearance). Maybe, for this reason, animals collected for museum storage are usually fixed in formalin [13]. Probably this possible effect had influence in the significant differences observed for the number of polychaetes between the preservation procedures, since more individuals were found in samples 

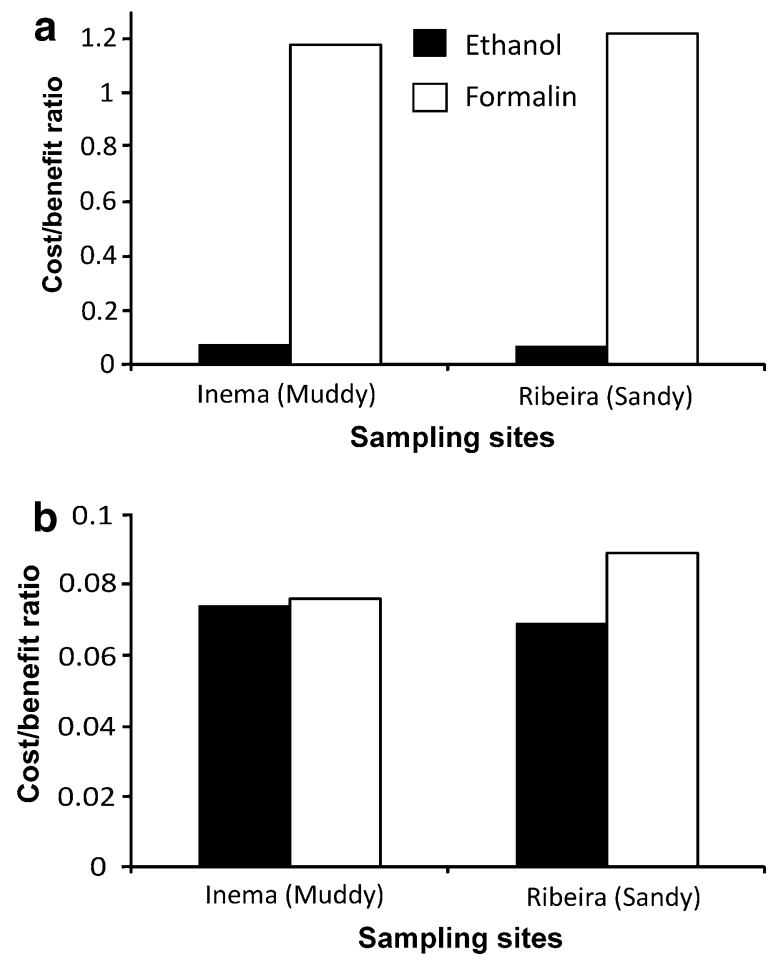

Fig. 4 Cost/benefit ratio of sample preservation procedures $\mathbf{a}$ with and $\mathbf{b}$ without fume hood costs

fixed with formalin. Such a difference was only observed in the sandy sediment site, possibly due to the smaller abundance collected on the muddy than the sandy sediment. The transition to the anoxic zone in muddy sediment occurs in a few centimeters from the surface, which restricts the distribution of some benthic species $[1,21]$. This characteristic may be associated to the lower number of specimens recorded in the muddy site.

Polychaetes, as well as other relatively fragile body taxa (e.g. Nemertea), are more susceptible to deformations in morphological structures following preservation procedures [2]. Costa-Paiva et al. [14] analyzed the effects of anesthesia and fixation on some morphological characters of the polychaete sabelliid Branchiomma luctuosum, and they observed that minor deformations were obtained with freshwater anesthesia or direct preservation in 70 or $100 \%$ ethanol. The same authors also pointed out that relatively fragile body animals are differently affected by preservation procedures. Future studies should carefully evaluate the effects of preservation procedures on morphological characters of different species of groups such as Polychaeta, focusing in time of preservation (i.e. days, weeks, months, and years).

Moreover, some attention should be given to anesthesia. This is a pre-fixation procedure mainly used in highly contractile animals, which is usually performed in a short time until animals are anesthetized or dead [2]. Anesthesia might possibly improve the outcome of both preservation methods evaluated in the present study, which would not be helpful in differentiating these methods. On the other hand, anesthesia could improve the outcome of only one procedure, and so would act as a confound variable for the present analysis. This possible scenario highlights the need of studies analyzing the interaction between anesthesia and following preservation procedures. Anyway, anesthesia is more commonly used in studies of specific taxonomic groups, such as polychaetes, or population studies.

Regarding the cost/benefit of preservation procedures, the separated analysis for each site was adopted due to spatial differences already presented. A well-marked difference between procedures was observed in both sediment types, where the best cost/benefit ratio was observed for $70 \%$ ethanol. As already stated, this result was more related to differences in the additional costs for formalin-fixed samples. According Wetzel et al. [13], the price difference between the two substances plays a considerable role if we take into account the additional costs for safety requirements of formalin handling and the differences in hazardous waste fees. In the present study, the hazardous waste fees where not analyzed, but the costs of safety equipment (i.e. mask, safety glasses, and fume hood) where included for formalin-fixed samples.

The safety requirements (i.e. lab coat, masks, gloves, safety glasses, fume hood) and care of the substance disposal are similarly recommended for both substances [see 22]. However, in benthic research labs all these equipment is generally used only when handling samples fixed in formalin. Samples preserved in ethanol are usually handled outside of fume hoods, only using gloves and lab coat. Anyway, we recommend all benthic researchers to follow the full safety procedure when handling samples with ethanol or formalin. There is an increased exposure through inhalation of ethanol or formalin vapors because labs are often poorly ventilated, leading to irritation of the upper respiratory tract and eyes. Thus, the personal protective equipment and fume hood are really necessary.

Many researchers had highlighted the special concern when using formalin due to imminent health and environmental risks [e.g. 13, 23]. Formalin vapors have irritating effects on mucous membranes, and contact with the skin results in dermatitis [24]. In addition, Coggon et al. [6] and Bosetti et al. [7] called attention to the higher risk of cancer in workers exposed to formalin (or formaldehyde). Furthermore, the use of formalin is not suitable for molecular research. These health risks of using formalin are important and should be considered in a cost/benefit analysis. However, their inclusion in the analysis becomes 
subjective since assigning a cost to these issues is quite tricky.

\section{Conclusion}

Studies about different sample preservation procedures are not as frequent as those evaluating other methodological aspects (e.g. sampling gear, mesh size and taxonomic resolution). Previously performed studies presented contradictory results, showing the need of a better guideline for the analysis of this issue. Assessments of the preservatives substances effects on biomass estimates have been widely explored. Nonetheless, assessments of preservative effects on quantitative data are apparently absent, and probably our study is pioneering in this regard so far. In the present study we did not observe significant differences between the preservation methods for macrofaunal assemblages. Polychaeta was the most affected group by the different procedures, presenting significantly higher abundances with formalin fixation. Thus, if a study is especially interested in polychaetes, we suggest the use of $10 \%$ formalin-fixed samples once these are properly handled. The use of ethanol without prior fixation had the best cost/benefit ratio, so its use is preferable for the study of the whole benthic community or assemblage. Further studies should evaluate how quantitative patterns are affected by: (1) exposure time of preservation; (2) anesthesia interaction; and (3) morphological deformations (e.g. impossibility of identification).

\begin{abstract}
Abbreviations
TSB: Todos os Santos Bay; PVC: polyvinyl chloride; PPE: personal protective equipment; E: ethanol; FE: formalin and ethanol; PERMANOVA: permutational multivariate analysis of variance; ANOVA: analysis of variance; MANOVA: multivariate analysis of variance; SNK: Student Newman-Keuls; nMDS: non-metric multidimensional scaling; CB: cost/benefit; $C t$ : total cost; $p$ : precision; $C_{u}$ : cost per unit; $C_{a}$ : additional cost; $S E$ : standard error; CFBio: Conselho Federal de Biologia (Federal Council of Biology); s: standard deviation; $n$ : sample size; $X$ : sample mean; NS: not significant; DF: degrees of freedom; MS: mean squares; $p$ (perm): permuted $p$ value; $p(\mathrm{MC})$ : Monte Carlo $p$ value.
\end{abstract}

\section{Authors' contributions}

Both authors carried out the collection, analysis and interpretation of data, as well as drafting and critical revision of the study. Both authors read and approved the final manuscript.

\section{Author details}

${ }_{1}^{1}$ Programa de Pós-Graduação em Ecologia, Laboratório de Biologia e Tecnologia Pesqueira, Departamento de Biologia Marinha, Centro de Ciências da Saúde, Instituto de Biologia, Universidade Federal do Rio de Janeiro, Av. Carlos Chagas Filho, 373, Bloco A, Ilha do Fundão, Rio de Janeiro, RJ CEP 21944-970, Brazil. ${ }^{2}$ Laboratório de Ecologia Bentônica, Instituto de Biologia, Universidade Federal da Bahia, Rua Barão de Geremoabo, s/n, Campus de Ondina, Salvador, Bahia CEP 40170-000, Brazil.

\section{Acknowledgements}

The authors would like to thanks the 'Programa de Pós Graduação em Ecologia e Biomonitoramento', from 'Universidade Federal da Bahia' (Bahia-Brazil), lecturers and students to their earlier discussions for the development of this study. Thanks also to Dr. P. L. B. Rocha (UFBA) and Dr. R. Coutinho (IEAPM) for their suggestions on this manuscript and to LEB (Laboratório de Ecologia
Bentônica) team for their help in the field. We also thank to J. Patterson for the assistance with the English language review. Finally, we thank the Editor and to the three anonymous referees for the corrections and valuable comments on the manuscript.

\section{Competing interests}

The authors declare that they have no competing interests.

\section{Availability of data and materials}

Data are available in a master degree dissertation at the repository from Federal University of Bahia ('Universidade Federal da Bahia'): http://repositorio. ufba.br/ri/handle/ri/14448.

\section{Consent for publication}

Not applicable.

\section{Ethics approval and consent to participate}

This article does not contain any studies with human participants performed by any of the authors. All applicable international, national, and/or institutional guidelines for the care and use of animals were followed.

\section{Funding}

This study was supported by CNPQ —-'Conselho Nacional de Desenvolvimento Científico e Tecnológico' (Grant Number 478265/2008-5). GBGS and FB were supported by a scholarship (CNPQ No. 565812/2008-4) and fellowships (PQ/ CNPQ No. 302642/2008-0; No. 303897/2011-2; No. 239978/2012-9) from CNPQ, respectively.

\section{Publisher's Note}

Springer Nature remains neutral with regard to jurisdictional claims in published maps and institutional affiliations.

Received: 8 June 2017 Accepted: 23 October 2017

Published online: 27 October 2017

\section{References}

1. Lenihan HS, Micheli F. Soft-sediment communities. In: Bertness MD Gaines SD, Hay ME, editors. Marine community ecology. Massachusetts: Sinauer Associates Inc; 2001. p. 253-87.

2. Lincoln RJ, Sheals GJ. Invertebrate animals: collection and preservation. London: Cambridge University Press; 1979.

3. Eleftheriou A, Moore DC. Macrofauna techniques. In: Eleftheriou A, Mcintyre A, editors. Methods for the study of marine benthos. 3rd ed. Oxford: Blackwell Science; 2005. p. 160-228.

4. Souza GBG. Avaliação de métodos aplicados ao estudo de assembleias macrozoobentônicas de substratos não consolidados. Salvador: Universidade Federal da Bahia (dissertation); 2010. http://repositorio.ufba.br/ri/ handle/ri/14448. Accessed 8 May 2017.

5. Thavarajah R, Mudimbaimannar VK, Elizabeth J, Rao UK, Ranganathan K. Chemical and physical basics of routine formaldehyde fixation. J Oral Maxillofac Pathol. 2012;16(3):400-5

6. Coggon D, Harris EC, Poole J, Palmer KT. Extended follow-up of a cohort of british chemical workers exposed to formaldehyde. J Natl Cancer Inst. 2003;95(21):1608-15

7. Bosetti C, Maclaughlin JK, Tarone RE, Pira E, Vecchia C. Formaldehyde and cancer risk: a quantitative review of cohort studies through 2006. Ann Oncol. 2008;19:24-43.

8. Howmiller RP. Effects of preservatives on weights of some common macrobenthic invertebrates. Trans Am Fish Soc. 1972;101(4):743-6.

9. Mills EL, Pittman K, Munroe B. Effect of preservation on the weight of marine benthic invertebrates. Can J Fish Aquat Sci. 1982;39:221-4.

10. Leuven RSEW, Brock TCM, Druten HAM. Effects of preservation on dryand ash-free dry weight biomass of some common aquatic macro-invertebrates. Hydrobiologia. 1985;127:151-9.

11. Gaston GR, Bartlett JHW, Mcallister AP, Heard RW. Biomass variations of estuarine macrobenthos preserved in ethanol and formalin. Estuaries. 1996;19(3):674-9. 
12. Von Schiller D, Solimini AG. Differential effects of preservation on the estimation of biomass of two common mayfly species. Arch Hidrobiol. 2005;164(3):325-34.

13. Wetzel MA, Leuchs $H$, Koop JHE. Preservation effects on wet weight, dry weight, and ash-free dry weight biomass estimates of four common estuarine macro-invertebrates: no difference between ethanol and formalin. Helgol Mar Res. 2005;59:206-13.

14. Costa-Paiva EM, Paiva PC, Klautau M. Anaesthetization and fixation effects on the morphology of sabellid polychaetes (Annelida: Polychaeta: Sabellidae). J Mar Biol Assoc UK. 2007;87:1127-32.

15. Cirano M, Lessa GC. Oceanographic characteristics of Baía de Todos os Santos, Brazil. Rev Bras Geofis. 2007;25(4):363-87.

16. Hurlbert SH. Pseudoreplication and the design of ecological field experiments. Ecol Monogr. 1984;54(2):187-211.

17. Anderson MJ. PERMANOVA: a FORTRAN computer program for permutational multivariate analysis of variance. New Zealand: Department of Statistics, University of Auckland; 2005.

18. Anderson MJ. A new method for non-parametric multivariate analysis of variance. Austral Ecol. 2001;26:32-46.
19. Souza GBG, Barros F. Analysis of sampling methods of estuarine benthic macrofaunal assemblages: sampling gear, mesh size, and taxonomic resolution. Hydrobiologia. 2015;743:157-74.

20. Andrew NL, Mapstone BD. Sampling and the description of spatial pattern in marine ecology. Oceanogr Mar Biol Annu Rev. 1987;25:39-90.

21. Gray JS, Elliot M. The ecology of marine sediments-from science to management. New York: Oxford University Press; 2009.

22. NIOSH-National Institute for Occupational Safety and Health. NIOSH Pocket Guide to Chemical Hazards-NPG. 2007. https://www.cdc.gov/ niosh/docs/2005-149/pdfs/2005-149.pdf. Accessed 10 Aug 2017.

23. Black AR, Dodson SI. Ethanol: a better preservation technique for Daphnia. Limnol Oceanogr Methods. 2003;1:45-50.

24. OSHA-Occupational Safety and Health Administration. Regulations (Standards - 29 CFR) — Substance technical guidelines for formalin. 2006. http://www.osha.gov/pls/oshaweb/owadisp.show_document?p_ table=STANDARDS\&p_id=10076. Accessed 10 May 2017.

\section{Submit your next manuscript to BioMed Central and we will help you at every step:}

- We accept pre-submission inquiries

- Our selector tool helps you to find the most relevant journal

- We provide round the clock customer support

- Convenient online submission

- Thorough peer review

- Inclusion in PubMed and all major indexing services

- Maximum visibility for your research

Submit your manuscript at www.biomedcentral.com/submit
() Biomed Central 